\title{
EXPRESSION OF E-CADHERIN AND BETA-CATENIN IN THE DEVELOPING LUNG AFTER PRENATAL ADMINISTRATION OF VITAMIN A
}

\author{
S. Santos ${ }^{1}$, R. Carvalho ${ }^{1}$, M. Pinto ${ }^{1}$, A.C. Coelho ${ }^{1}$, C. Gonçalves ${ }^{2}$, V. Bairos ${ }^{2}$ \\ ${ }^{1}$ Universidade de Trás-os-Montes e Alto Douro, Vila Real, ${ }^{2}$ Faculdade de Medicina - Universidade de \\ Coimbra, Coimbra, Portugal
}

Cadherin-catenin complexes stabilize adhesive contacts between the neighboring cells, direct actin cytoskeleton reorganization, and are tightly linked to several key signal transduction networks, which makes them crucial players during lung morphogenesis. Vitamin A ant its derivatives (retinoids) are potent regulators of cell proliferation and differentiation essential for normal lung development. In order to investigate vitamin A influence on E-cadherin and beta-catenin during lung development we conducted an in vivo study in which pregnant mice were subjected to subcutaneous administration of vitamin A on the 12th gestational day. The lungs from control and treated animals were collected daily from the 15th gestational day till the 3th day of life and processed for routine immunohistochemistry. According to our results, betacatenin expression was not changed by vitamin A administration, while the expression of E-cadherin increased. E-cadherin expression was significantly higher in the lungs of vitamin A-treated animals at the $15^{\text {th }}$ and $18^{\text {th }}$ gestational days, at the day of birth and at the first and second day of life. Our previous works showed that prenatal administration of vitamin A modified branching morphogenesis resulting in a more mature lung at the canalicular and subsequent stages of lung development, a feature that can be explained by its influence on the regulation of the correct size and shape of the epithelial growing buds in which the Ecadherin spatial and temporal expression is of pivotal importance. 